\title{
Ueber die Ablenkung der Lothlinie in grossen Höhen.
}

Dass in grossen Höben über der Erdoberfläche die Lothlinie eine Ablenkung zeigen müsse, verglichen mit derjenigen Richtung, welche sie in derselben Normale des Erdsphäroids an der Erdoberfläche besitzt, folgt einfach aus der Thatsache, dass in grossen Höhen die Anziehung der Erde abnimmt, die Centrifugalkraft dagegen zunimmt. Denn danach muss die Resultante beider, d. i. die Schwere, in grossen Höhen (ausgenommen am Aequator und in den Polen) einen kleineren Winkel mit der Erdachse einschliessen als in derselben Normale am Erdboden, oder die.Polhähe muss dort zu gross erscheinen. Ueber die Grösse dieser Ablenkung aber dürfte es zur Zeit unnöglieh sein eine vollkommen exacte Bestimmung zu treffen. Die Annahme des Erdsphäroides als eines h omogenen Körpers widerstreitet zu sehr den bekannten geognostischen Thatsachen, auch liefert sie eine Erdgestalt, die nur wenig mit den Ergebnissen geodätischer Operationen harmonirt. Die Annahme eines nicht homogenen Erdkörpers aber scheitert an der Unmöglichkeit, ein Gesetz aufustellen, nach welchem die Dichtigkeit mit der Tiefe zunimmt. Nichts desto weniger -scheint an dem folgenden Wege zum wenigsten eine angenäherte Bestimmung der gedachten Grösse erreichbar zu sein.

Wir werden, um grösserer Deutlichkeit willen, streng zwischen "Anziehung" und "Schwere" unterscheiden, indem wir unter Schwere stets nur die Resultante aus Anziehung and Centrifugalkraft verstehen.

1) Es sei $a$ die grosse Halbachse des Erdsphäroids. In irgend einem Punkte des Aequators sei ferner $g_{0}$ die Schwere, $G_{0}$ die Anziehung. Für die Centrifugalkraft am Aequator hat man den bekannten Ausdruck

$$
\frac{4 \pi^{2} a}{T^{2}}
$$

-wo $I$ ' die Dauer des Sterntages in Secanden bedeutet, oder wenn man setzt

$$
\frac{4 \pi^{2}}{T^{2}}=f
$$

so wird fa der Ausdruck fïr die Centrifugalkraft am Aequator. Aus der Zusammensetzung der Kräfte folgt sodann

$$
\begin{aligned}
g_{0} & =G_{0}-f a \\
\text { oder wenn } \operatorname{man} \frac{f a}{G_{0}} & =\propto \operatorname{setzt} \\
g_{0} & =G_{0}(1-\alpha) \ldots \ldots \ldots \ldots(1) \ldots \ldots
\end{aligned}
$$

Die Beobachtungen geben $g_{0}=9^{\mathrm{m}} 78061$ und mit Rücksicht auf die Bessel'schen Erddimensionen wird $f a=0^{\mathrm{m}} 03391$ also $G_{0}=9^{\mathrm{m}} 81452$. Folglich hat man für das Verhältniss der Centrifugalkraft zur Anziehung am Aequator den Werth

$$
\alpha=\frac{1}{289,42} \ldots \ldots \ldots \ldots \ldots(2) .
$$

und für das Verhältniss der Centrifugalkraft zur Schwere am Aequator den Werth

$$
\frac{\alpha}{1-\alpha}=\beta=\frac{1}{288,42} \ldots \ldots \ldots \ldots
$$

Die Grössen $\alpha$ und $\beta$ sind offenbar von derselben Ordnung wie die Abplattung des Erdsphäroids, welche wir hier als die erste Ordnung bezeichnen wollen.

2) In irgend einem Punkte $A$ der Erdoberfläche von der Polhöhe $\varphi$ sei $g$ die Schwere, $G$ die Anziehung. Die Richtung der Anziebung schneide die Erdachse im Punkte $C$, und es sei $A C=r$ und der Winkel, den diese Linie mit der Ebene des Aequators einschliesst, $=\psi$. Für die Centrifugalkraft im Punkte $A$ kann man sodann setzen $f r \cos \psi$. Da un in diesem Punkte $A$ die Richtung der Schwere mit der Normale des Erdsphäroids zusammenfällt, so folgt aus der Zusammensetzung der Kräfte

d. i.

$$
\operatorname{tang} \varphi=\frac{G \sin \psi}{G \cos \psi-f r \cos \psi}
$$

$$
\operatorname{tang} \varphi=\frac{\operatorname{tang} \psi}{1-\alpha} \ldots \ldots \ldots \ldots
$$

wo wir der Kürze wegen den Beweis unterdrücken, dass bis auf Grössen, welche gegen $\propto$ verschwinden,

gesetzt werden kann.

$$
\frac{f r}{G}=\frac{f a}{G_{0}}=\alpha
$$

Die Winkel $\varphi$ und $\psi$ stehen hiernach in einer ähnlichen Beziehung zu einander wie geographische und geocentrische Breite. Jedoch ist $\psi$ immer grösser als die geocentrische Breite, oder die Richtung der Anziehung geht zwischen der -Normale und dem Mittelpunkte des Ellipsoids hindurch, wie es auch beim homogenen Ellipsoid der Fall ist.

$$
\text { Für } \varphi=45^{\circ} \text { ist } \psi=44^{\circ} 54^{\prime} 4^{\prime \prime} 6 \text {. }
$$

3) Im Punkte $A$ von der Polhöhe $\varphi$ errichte man auf der Erdoberfläche eine Normale $A B$ und setze die Höhe $A \dot{B}=h$. Zur Bestimnung der Anziehung in $B$ mũssten wir, unter der Voraussetzung eines homogenen Ellipsoids, nach dem Satze von Ivory verfahren. Da aber die Hypothese der Homogeneität nicht der Wirklichkeit entspricht, überdies wir uns nur auf kleine Werthe von $h$ beschränken, so erscheint es ausreichend, für den genannten $Z$ week einfach den vorhin bestimmten Punkt $\boldsymbol{C}$ der Erdachse wie den anziehenden Punkt gelten zu lassen. Dies wird nicht nur der Wabrheit nahe bleiben, sondern es giebt auch eine ziemlieh einfache Rechnung. 
Denn es sei $B C=r^{\prime}$ und der Winkel, den diese Linie mit der Ebene des Aequators einschliesst, $=\psi^{\prime}$. Dann hat díe Anziehung im Punkte $B$ den Werth $G \frac{r^{2}}{r^{2}}$, die Centrifugalkraft im Punkte $B$ wird $f r^{\prime} \cos \psi^{\prime}$, und wenn man den Winkel, den die Riehtung der Schwere im Punkte $B$ mit der Ebene des Aequators hildet, mit $\varphi^{\prime}$ bezeichnet, so folgt aus der Zusammensetzung der Kräfte

$$
\operatorname{tang} \varphi^{\prime}=\frac{G \frac{r^{2}}{r^{2}} \sin \psi^{\prime}}{G \frac{r^{2}}{r^{2}} \cos \psi^{\prime}-f r^{\prime} \cos \psi^{\prime}}
$$

d. 1 .

$$
\operatorname{tang} \varphi^{\prime}=\frac{\operatorname{tang} \psi^{\prime}}{1-\frac{\alpha r^{33}}{r^{3}}} \ldots \ldots \ldots \ldots \ldots \ldots \ldots(5)
$$

Zur Bestimmung von $r^{\prime}$ und $\psi^{\prime}$ durch $h$ bat man überdies die Gleichungen

àus denen folgt

$$
\begin{aligned}
& r^{\prime} \sin \psi^{\prime}=r \sin \psi+h \sin \varphi \\
& r^{\prime} \cos \psi^{\prime}=r \cos \psi+h \cos \varphi
\end{aligned}
$$

$$
\begin{aligned}
& r^{\prime 2}=r^{2}+h^{2}+2 r h \cos (\varphi-\psi) \ldots \ldots \ldots \\
& \operatorname{tang} \psi^{\prime}=\frac{r \sin \psi+h \sin \varphi}{r \cos \psi+h \cos \phi} \ldots \ldots \ldots
\end{aligned}
$$

Soll nun hieraus derWerth von $\varphi^{\circ}-\phi$ für kleine Werthe von $h$, d. $h$. die Ablenkung der Lothlinie in der Höhe $h$ gefunden werden, so bestimme man aus (5), (6), (7) den

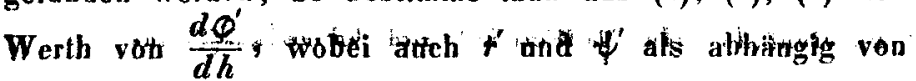
$h$ anzusehen sind, und setze hinterher $h=0$, folglich auch $r^{\prime}=r$ und $\psi^{\prime}=\psi$. Diese Rechnung giebt, bis auf Grössen der zweiten Ordnung genau, für die Ablenkung $d \varphi^{\prime}$ in der Höhe $d h$ den Ausdruck

$$
d \varphi^{\circ}=\frac{2 \alpha \sin 2 \varphi}{1-\alpha}\left(1-\frac{1}{4} \alpha \sin \varphi^{2}\right) \frac{d h}{r} \ldots \ldots(8)
$$

wofür man auch, ohne merklichen Fehler, mit Weglassung der Klammer noch einfacher schreiben kann

$$
d \varphi^{\prime}=2 \beta \sin 2 \varphi \cdot \frac{d h}{r} \ldots \ldots \ldots(9)
$$

und worin für $r$ der Halbmesser des Aequators gesetzt werden darf.

Vm (Wófür nán jetzt auch $d \varphi$ schreiben kann) in Seeunden ausgedräckt gu erbalten, muss die rechte Seite dieserer Glèichung noch mit dem Factor 206264,8 multiplicirt werden.

Der Arsidruck (9) wird ein Maximum für $\varphi=45^{\circ}$. Soll in diesem Falle die Ablenkung $d \varphi=1$ Secunde sein, so muss man haben $d h=4460^{\mathrm{m}}=13730$ Pariser Fuss, welche Höhe die höchsten Bergspitzen der Schweiz übertrifft, den Niontblanc aber nicht erreicht.

Zur Vergleichung mag bemerkt werden, dass in einem b omogenen Sphäroid von den Dimensionen der Erde die Richtungen der Anziehung in $A$ und $B$, beide Punkte einander sehr nahe liegeod vorausgesetzt, sich schon schneiden, bevor sie die Erdachse treffen, uघd dass demgemäss der Werth $d \varphi$ in dem Verhältniss $1: 1,14$ grösser ausfällt als in (9). Hiernach würde, unter $45^{\circ}$ Polhöhe, eine Ablenkung von 1 Secunde schon in der Höhe von $3910^{\mathrm{m}}=12040$ Pariser Fuss eintreten, welche Höhe die höchsten Spitzew der Schweiz nicht völlig erreicht.

4) Aus allem Vorstehenden folgt, dass die wahre Lothlinie eines gegebenen Punkts $A$ der Erdoberfläche (ausgenommen an Aequator und id den Polen) eine krumme Linie ist, welche in der Meridian-Ebene des gegebenen Punkts liegt und ihre concave Seite nach dem nächsten Erdpol hiowendet. Die Tangente dieser krummen Linie in irgend einem Punkte derselben stellt die Richtung der Schwere in diesem Punkte dar. Die Diffentialgleichung der Lothlinie ist also in der Gleichung (5) enthalten, wo $r^{\prime}$ und $\psi^{\prime}$ wie die veränderlichen Coordinaten auzusehen sind. Dann setzt man darin, un rechtwinkelige Coordinaten einzuführen, $r^{\prime} \cos \psi^{\prime}=x$ un'd $r^{\prime} \sin \psi^{\prime}=y$, so wird $\operatorname{tang} \varphi^{\prime}=\frac{d y}{d x}$ und die Gleichung nimmt die Gestalt an

$$
\frac{d y}{d x}=\frac{y}{x} \cdot \frac{1}{1-\frac{\alpha}{r^{3}}\left(x^{2}+y^{2}\right)^{\frac{3}{2}}} \ldots \ldots \ldots(10)
$$

Diese Gleichung kann aber auch auf dem folgenden Wege erhalten werden, der zugleich für die ganze'vorstehende Entwickelung eine bemerkenswerthe Rechnungsprobe bietet. Laplace leitet in der Mécanique céleste (3. Buch, 7. Cap.) für geringe Erhebungen über der Erdoberfläcbe die Gleichung einer Surface de niveau $a b$, d. h. einer Fläche, in deren sämmtlichen Punkten die Richtung der Schwere auf ihr senkrecht steht. Diese Gleichung hat in der hier gebrauchten Bezeichnung die Gestalt

$$
\text { Const. }=\frac{2 r}{r^{\prime}}+\frac{\alpha^{\prime} r^{\prime 2}}{r^{2}} \cos \psi^{\prime 2}
$$

Lässt man darin die Constante sich ändern, so stellt offenbar die rechtwinkelige Trajectorie aller dieser Flăchen die in Rede stehende Lothlinie dar. In der That giebt die Ausfuhrung dieser Rechnung, bei der man sich auf eine MeridianEbene beschränken darf, genau wieder die Gleichung (10).

Hannover, Juli 1869. 